\title{
MORE ON RC-LINDELÖF SETS AND ALMOST RC-LINDELÖF SETS
}

\author{
MOHAMMAD S. SARSAK
}

Received 25 November 2004; Revised 3 April 2006; Accepted 30 May 2006

We study new properties and characterizations of rc-Lindelöf sets and almost rc-Lindelöf sets; a special interest is given to the mapping properties of such sets. We also obtain some product theorems concerning rc-Lindelöf spaces.

Copyright (c) 2006 Hindawi Publishing Corporation. All rights reserved.

\section{Introduction and preliminaries}

A subset $A$ of a space $X$ is called regular open if $A=\operatorname{Int} \bar{A}$, and regular closed if $X \backslash A$ is regular open, or equivalently, if $A=\overline{\operatorname{Int} A}$. $A$ is called semiopen [16] (resp., preopen [17], semi-preopen [3], $b$-open [4]) if $A \subset \overline{\operatorname{Int} A}$ (resp., $A \subset \operatorname{Int} \bar{A}, A \subset \overline{\operatorname{Int} \bar{A}}, A \subset \overline{\operatorname{Int} A} \cup \operatorname{Int} \bar{A}$ ). The concept of a preopen set was introduced in [6] where the term locally dense was used and the concept of a semi-preopen set was introduced in [1] under the name $\beta$-open. It was pointed out in [3] that $A$ is semi-preopen if and only if $P \subset A \subset \bar{P}$ for some preopen set $P$. Clearly, every open set is both semiopen and preopen, semiopen sets as well as preopen sets are $b$-open, and $b$-open sets are semi-preopen. $A$ is called semiclosed (resp., preclosed, semi-preclosed, $b$-closed) if $X \backslash A$ is semiopen (resp., preopen, semi-preopen, $b$-open). $A$ is called semiregular [8] if it is both semiopen and semiclosed, or equivalently, if there exists a regular open set $U$ such that $U \subset A \subset \bar{U}$.

Clearly, every regular closed (regular open) set is semiregular. The semiclosure (resp., preclosure, semi-preclosure, $b$-closure) denoted by $\operatorname{scl} A$ (resp., $\operatorname{pcl} A, \operatorname{spcl} A, \operatorname{bcl} A$ ) is the intersection of all semiclosed (resp., preclosed, semi-preclosed, $b$-closed) subsets of $X$ containing $A$, or equivalently, is the smallest semiclosed (resp., preclosed, semi-preclosed, $b$-closed) set containing $A$. Dually, the semi-interior (resp., preinterior, semi-preinterior, $b$-interior) denoted by $\operatorname{sint} A$ (resp., $\operatorname{pint} A$, spint $A$, $\operatorname{bint} A$ ) is the union of all semiopen (resp., preopen, semi-preopen, $b$-open) subsets of $X$ contained in $A$, or equivalently, is the largest semiopen (resp., preopen, semi-preopen, $b$-open) set contained in $A$.

A function $f$ from a space $X$ into a space $Y$ is called almost open [20] if $f^{-1}(\bar{U}) \subset$ $\overline{f^{-1}(U)}$ whenever $U$ is open in $Y$, semicontinuous [16] if the inverse image of each 
open set is semiopen, $\beta$-continuous [1] if the inverse image of each open set is $\beta$-open, weakly $\theta$-irresolute [13] if the inverse image of each regular closed set is semiopen, rccontinuous [14] if the inverse image of each regular closed set is regular closed, and wrccontinuous [2] if the inverse image of each regular closed set is semi-preopen. We will use the term semiprecontinuous to indicate $\beta$-continuous. Clearly, every semicontinuous function is semi-precontinuous, every rc-continuous function is weakly $\theta$-irresolute, and every weakly $\theta$-irresolute function is wrc-continuous. It is also easy to see that a function that is both semicontinuous (resp., semi-precontinuous) and almost open is weakly $\theta$-irresolute (resp., wrc-continuous).

A function $f$ from a space $X$ into a space $Y$ is called somewhat continuous [12] if for each nonempty open set $V$ in $Y$, int $f^{-1}(V) \neq \phi$.

A space $X$ is called a weak $P$-space [18] if for each countable family $\left\{U_{n}: n \in \mathbb{N}\right\}$ of open subsets of $X, \overline{\cup U_{n}}=\cup \bar{U}_{n}$. Clearly, $X$ is a weak $P$-space if and only if the countable union of regular closed subsets of $X$ is regular closed (closed).

A space $X$ is called rc-Lindelöf [15] (resp., nearly Lindelöf [5]) if every regular closed (resp., regular open) cover of $X$ has a countable subcover, and called almost rc-Lindelöf [10] if every regular closed cover of $X$ has a countable subfamily whose union is dense in $X$.

A subset $A$ of a space $X$ is called an $S$-set in $X$ [7] if every cover of $A$ by regular closed subsets of $X$ has a finite subcover, and called an rc-Lindelöf set in $X$ (resp., an almost rcLindelöf set in $X$ ) [9] if every cover of $A$ by regular closed subsets of $X$ admits a countable subfamily that covers $A$ (resp., the closure of the union of whose members contains $A$ ). Obviously, every $S$-set is an rc-Lindelöf set and every rc-Lindelöf set is an almost rcLindelöf set; it is also clear that a subset $A$ of a weak $P$-space $X$ is rc-Lindelöf in $X$ if and only if it is almost rc-Lindelöf in $X$.

Throughout this paper, $\mathbb{N}$ denotes the set of natural numbers. For the concepts not defined here, we refer the reader to Engelking [11].

In concluding this section, we recall the following facts for their importance in the material of our paper.

Theorem 1.1 [9]. If $A$ is an $r c$-Lindelöf (resp., almost rc-Lindelöf) set in a space $X$ and $B$ is a regular open subset of $X$, then $A \cap B$ is rc-Lindelöf (resp., almost rc-Lindelöf) in $X$. In particular, a regular open subset $A$ of an rc-Lindelöf (resp., almost rc-Lindelöf) space $X$ is rc-Lindelöf (resp., almost rc-Lindelöf) in X.

Theorem 1.2 [9]. Let $A$ be a preopen subset of a space $X$ and $B \subset A$. Then $B$ is $r$-Lindelöf (resp., almost $r c$-Lindelöf) in $X$ if and only if $B$ is $r c$-Lindelöf (resp., almost rc-Lindelöf) in A. In particular, a preopen subset $A$ of a space $X$ is $r c$-Lindelöf (resp., almost rc-Lindelöf) in $X$ if and only if $A$ is an rc-Lindelöf (resp., almost rc-Lindelöf) subspace.

Proposition 1.3 [19]. If $A$ is an almost $r$-Lindelö set in a space $X$ and $A \subset B \subset \bar{A}$, then $B$ is almost $r$-Lindelöf in $X$.

Proposition 1.4 [9]. The countable union of rc-Lindelöf (resp., almost rc-Lindelöf) sets in a space $X$ is rc-Lindelöf (resp., almost rc-Lindelöf) in $X$. 
Proposition 1.5 [9]. A subset $A$ of a space $X$ is rc-Lindelöf (resp., almost rc-Lindelöf) in $X$ if and only if every cover of $A$ by semiopen subsets of $X$ admits a countable subfamily the union of the closures of whose members (resp., the closure of the union of whose members) contains A.

Proposition 1.6 [19]. Let $A$ be a preopen, almost $r c$-Lindelöf set in a space $X$ and $B$ a regular closed subset of $X$, then $A \cap B$ is almost $r c$-Lindelö in $X$. In particular, a regular closed subset $A$ of an almost $r c$-Lindelöf space $X$ is almost rc-Lindelöf in X.

Lemma 1.7. If $A$ is a preopen subset of a space $X$ and $U$ is open in $X$, then $\overline{A \cap U} \cap A=$ $\bar{U} \cap A$.

\section{Further properties}

This section is devoted to study new properties concerning rc-Lindelöf sets and almost rc-Lindelöf sets. We obtain several characterizations of rc-Lindelöf sets and almost rcLindelöf sets.

The following proposition is an improvement of Proposition 1.6 and the fact of Theorem 1.1 that a regular open subset of an almost rc-Lindelöf space $X$ is almost rcLindelöf in $X$.

Proposition 2.1. Let $A$ be a preopen, almost $r c$-Lindelöf set in a space $X$ and $B$ a semiregular subset of $X$, then $A \cap B$ is almost rc-Lindelof in $X$. In particular, a semiregular subset $A$ of an almost $r c$-Lindelöf space $X$ is almost $r c$-Lindelöf in $X$.

Proof. Since $B$ is a semiregular subset of $X$, there exists a regular open subset $U$ of $X$ such that $U \subset B \subset \bar{U}$, thus by Lemma 1.7, it follows that $A \cap U \subset A \cap B \subset \bar{U} \cap A \subset \overline{A \cap U}$. Since $A$ is almost rc-Lindelöf set in X, it follows from Theorem 1.1 that $A \cap U$ is almost rc-Lindelöf set in $X$. The result yields from Proposition 1.3.

Proposition 2.2 [19]. If $A$ is a regular closed subset of a space $X$ such that $A$ is almost $r c$-Lindelöf in $X$, then $A$ is an almost rc-Lindelöf.

The following proposition includes an improvement of Proposition 2.2.

Proposition 2.3. Let $A$ be a semiopen subset of a space $X$ and $B \subset A$. If $B$ is rc-Lindelöf (resp., almost $r c$-Lindelöf) in $X$, then $B$ is $r c$-Lindelöf (resp., almost $r c$-Lindelöf) in $A$. In particular, if $A$ is a semiopen subset of a space $X$ such that $A$ is rc-Lindelöf (resp., almost rc-Lindelöf) in X, then $A$ is an rc-Lindelöf (resp., almost rc-Lindelöf) subspace.

Proof. Follows from Proposition 1.5 and the fact that if $A$ is a semiopen subset of a space $X$ and $B$ is semiopen in $A$, then $B$ is semiopen in $X$.

Corollary 2.4 [2]. Let $X$ be an rc-Lindelöf weak P-space. If $U \subset A \subset \bar{U}$, where $U$ is a regular open subset of $X$, then $A$ is an $r c$-Lindelöf subspace.

Proof. By Theorem 1.1, $U$ is an rc-Lindelöf set in $X$ and thus almost rc-Lindelöf in $X$. By Proposition 1.3, $A$ is almost rc-Lindelöf in $X$, but $X$ is a weak $P$-space, so $A$ is rcLindelöf in $X$. Finally, since $A$ is semiopen (it is moreover semiregular), it follows from Proposition 2.3 that $A$ is an rc-Lindelöf subspace. 
The following theorem includes new characterizations of rc-Lindelöf sets and almost rc-Lindelöf sets.

Theorem 2.5. Let $A$ be a subset of a space $X$. Then the following are equivalent.

(i) $A$ is rc-Lindelöf (resp., almost $r c$-Lindelöf) in $X$.

(ii) Every cover of A by semi-preopen subsets of $X$ admits a countable subfamily the union of the closures of whose members (resp., the closure of the union of whose members) contains A.

(iii) Every cover of A by b-open subsets of $X$ admits a countable subfamily the union of the closures of whose members (resp., the closure of the union of whose members) contains $A$.

(iv) Every cover of $A$ by semiopen subsets of $X$ admits a countable subfamily the union of the closures of whose members (resp., the closure of the union of whose members) contains $A$.

(v) Every cover of A by semiregular subsets of $X$ admits a countable subfamily the union of the closures of whose members (resp., the closure of the union of whose members) contains A.

Proof. (i) $\Rightarrow$ (ii): follows since the closure of a semi-preopen set is regular closed.

(ii) $\Rightarrow$ (iii) $\Rightarrow($ iv $) \Rightarrow(v) \Rightarrow($ i): follows from the following implications: regular closed $\Rightarrow$ semiregular $\Rightarrow$ semiopen $\Rightarrow b$-open $\Rightarrow$ semi-preopen.

The following theorem also characterizes rc-Lindelöf sets and almost rc-Lindelöf sets, it is a direct consequence of Theorem 2.5 and the definition of rc-Lindelöf (almost rcLindelöf) sets.

Theorem 2.6. Let $A$ be a subset of a space $X$. Then the following are equivalent.

(i) $A$ is rc-Lindelöf (resp., almost rc-Lindelöf) in X.

(ii) If $U_{\sim}=\left\{U_{\alpha}: \alpha \in \Lambda\right\}$ is a family of regular open subsets of $X$ satisfying that for any countable subcollection $U_{\sim}^{*}$ of $U_{\sim}, A \cap\left(\cap U_{\sim}^{*}\right) \neq \phi$ (resp., $\left.A \cap \operatorname{int}\left(\cap U_{\sim}^{*}\right) \neq \phi\right)$, then $A \cap\left(\cap U_{\sim}\right) \neq \phi$.

(iii) If $U_{\sim}=\left\{U_{\alpha}: \alpha \in \Lambda\right\}$ is a family of semi-preclosed subsets of $X$ satisfying that for any countable subcollection $U_{\sim}^{*}$ of $U_{\sim}, A \cap\left(\cap\left\{\right.\right.$ int $\left.\left.U: U \in U_{\sim}^{*}\right\}\right) \neq \phi$ (resp., $A \cap$ $\left.\operatorname{int}\left(\cap U_{\sim}^{*}\right) \neq \phi\right)$, then $A \cap\left(\cap U_{\sim}\right) \neq \phi$.

(iv) If $U_{\sim}=\left\{U_{\alpha}: \alpha \in \Lambda\right\}$ is a family of $b$-closed subsets of $X$ satisfying that for any countable subcollection $U_{\sim}^{*}$ of $U_{\sim}, A \cap\left(\cap\left\{\operatorname{int} U: U \in U_{\sim}^{*}\right\}\right) \neq \phi$ (resp., $A \cap \operatorname{int}\left(\cap U_{\sim}^{*}\right) \neq$ $\phi)$, then $A \cap\left(\cap U_{\sim}\right) \neq \phi$.

(v) If $U_{\sim}=\left\{U_{\alpha}: \alpha \in \Lambda\right\}$ is a family of semiclosed subsets of $X$ satisfying that for any countable subcollection $U_{\sim}^{*}$ of $U_{\sim}, A \cap\left(\cap\left\{\operatorname{int} U: U \in U_{\sim}^{*}\right\}\right) \neq \phi\left(\right.$ resp., $A \cap \operatorname{int}\left(\cap U_{\sim}^{*}\right)$ $\neq \phi)$, then $A \cap\left(\cap U_{\sim}\right) \neq \phi$.

(vi) If $U_{\sim}=\left\{U_{\alpha}: \alpha \in \Lambda\right\}$ is a family of semiregular subsets of $X$ satisfying that for any countable subcollection $U_{\sim}^{*}$ of $U_{\sim}, A \cap\left(\cap\left\{\operatorname{int} U: U \in U_{\sim}^{*}\right\}\right) \neq \phi$ (resp., $A \cap \operatorname{int}\left(\cap U_{\sim}^{*}\right)$ $\neq \phi)$, then $A \cap\left(\cap U_{\sim}\right) \neq \phi$.

\section{Invariance properties}

In this section, we mainly study several types of functions that preserve the property of being an rc-Lindelöf (almost rc-Lindelöf) set. 
Definition 3.1 [19]. A function $f$ from a space $X$ into a space $Y$ is said to be slightly continuous if $f(\bar{U}) \subset \overline{f(U)}$ whenever $U$ is open in $X$.

In [19], it was shown that if a function $f: X \rightarrow Y$ is slightly continuous and weakly $\theta$-irresolute, then $f(A)$ is almost rc-Lindelöf in $Y$ whenever $A$ is almost rc-Lindelöf set in $X$. The following theorem is analogous to this result; it has a similar proof that we will mention for the convenience of the reader.

THeORem 3.2. Let $f: X \rightarrow Y$ be a slightly continuous and weakly $\theta$-irresolute function. If $A$ is rc-Lindelöf set in $X$, then $f(A)$ is rc-Lindelöf in $Y$.

Proof. Let $\left\{U_{\alpha}: \alpha \in \Lambda\right\}$ be a cover of $f(A)$ by regular closed subsets of $X$. Then $\left\{f^{-1}\left(U_{\alpha}\right)\right.$ : $\alpha \in \Lambda\}$ is a cover of $A$ by semiopen subsets of $X$ (as $f$ is weakly $\theta$-irresolute). Since $A$ is rc-Lindelöf in $X$, it follows from Proposition 1.5 that there exist $\alpha_{1}, \alpha_{2}, \ldots \in \Lambda$ such that $A \subset \bigcup_{i=1}^{\infty} \overline{f^{-1}\left(U_{\alpha_{i}}\right)}$. For each $i \in \mathbb{N}$, there is an open subset $V_{i}$ of $X$ such that $V_{i} \subset$ $f^{-1}\left(U_{\alpha_{i}}\right) \subset \bar{V}_{i}$ and thus $\bigcup_{i=1}^{\infty} \overline{f^{-1}\left(U_{\alpha_{i}}\right)}=\bigcup_{i=1}^{\infty} \overline{V_{i}}$. Since $f$ is slightly continuous, it follows that $f(A) \subset \bigcup_{i=1}^{\infty} \overline{f\left(V_{i}\right)} \subset \bigcup_{i=1}^{\infty} \overline{U_{\alpha_{i}}}=\bigcup_{i=1}^{\infty} U_{\alpha_{i}}$. Hence $f(A)$ is rc-Lindelöf in $Y$.

Corollary 3.3. Let $f: X \rightarrow Y$ be a slightly continuous, semicontinuous, and almost open function. If $A$ is rc-Lindelöf (resp., almost rc-Lindelöf) in $X$, then $f(A)$ is rc-Lindelöf (resp., almost rc-Lindelöf) in $Y$.

Corollary 3.4. Let $f: X \rightarrow Y$ be a surjective, slightly continuous, semicontinuous, and almost open function. If $X$ is rc-Lindelöf, then $Y$ is rc-Lindelöf.

It will be seen later that the condition slightly continuous of Corollary 3.4 is not essential for preserving the almost rc-Lindelöf property.

Corollary 3.5 [2]. Let $f: X \rightarrow Y$ be a surjective, continuous, and almost open function. If $X$ is rc-Lindelöf, then $Y$ is rc-Lindelöf.

Obviously, every continuous function is both semicontinuous and slightly continuous. However, the converse is not true as the following example tells.

Example 3.6. Let $X=\{a, b, c\}, \tau=\{X, \phi,\{a\}\}, \tau^{*}=\{X, \phi,\{a, b\}\}$. Then the identity function from $(X, \tau)$ onto $\left(X, \tau^{*}\right)$ is a semicontinuous, slightly continuous, and almost open surjection. However, it is not continuous.

Proposition 3.7. Let $f: X \rightarrow Y$ be a semicontinuous function. If $X$ is extremally disconnected (i.e., every regular closed subset of $X$ is open), then $f$ is slightly continuous.

Proof. Let $U$ be open in $X$. Then $\operatorname{scl}(U)=U \cup \operatorname{int} \bar{U}=\bar{U}$ (as $X$ is extremally disconnected). Since $f$ is semicontinuous, it follows that $f(\operatorname{scl}(U))=f(\bar{U}) \subset \overline{f(U)}$. Hence $f$ is slightly continuous.

The following corollary is an immediate consequence of Corollary 3.4 and Proposition 3.7 .

Corollary 3.8 [2]. Let $f: X \rightarrow Y$ be a semicontinuous, almost open surjection, where $X$ is extremally disconnected. If $X$ is rc-Lindelöf, then $Y$ is rc-Lindelöf. 
The following example shows that if $X$ is extremally disconnected and $f: X \rightarrow Y$ is slightly continuous, almost open surjection, then $f$ need not be semicontinuous.

Example 3.9. Let $X=\{a, b, c\}, \tau=\{X, \phi,\{a, b\}\}, \tau^{*}=\{X, \phi,\{a\}\}$. Then $(X, \tau)$ is extremally disconnected, also the identity function from $(X, \tau)$ onto $\left(X, \tau^{*}\right)$ is slightly continuous and almost open ; it is, however, not semicontinuous.

Proposition 3.10 [10]. (i) Let $f: X \rightarrow Y$ be a somewhat continuous and weakly $\theta$-irresolute function. If $X$ is almost $r c$-Lindelöf, then $Y$ is almost $r c$-Lindelöf.

(ii) Let $f: X \rightarrow Y$ be a surjective, semicontinuous, and weakly $\theta$-irresolute function. If $X$ is almost $r c$-Lindelöf, then $Y$ is almost $r c$-Lindelöf.

Corollary 3.11. Let $f: X \rightarrow Y$ be a surjective, semicontinuous, and almost open function. If $X$ is almost rc-Lindelöf, then $Y$ is almost rc-Lindelöf.

The following corollary is an immediate consequence of Corollary 3.11 and the fact that for a weak $P$-space, the concepts of being rc-Lindelöf and almost rc-Lindelöf coincide.

Corollary 3.12 [2]. Let $f: X \rightarrow Y$ be a surjective, semicontinuous, and almost open function, where $Y$ is a weak $P$-space. If $X$ is rc-Lindelöf, then $Y$ is rc-Lindelöf.

Definition 3.13. A function $f: X \rightarrow Y$ is said to be somewhat precontinuous if for each nonempty open set $V$ in $Y$, pint $f^{-1}(V) \neq \phi$.

Remark 3.14. It was pointed out in [10] that every surjective semicontinuous function is somewhat continuous, a similar result that may be pointed out here asserts that every surjective semi-precontinuous function is somewhat precontinuous. However, the converses of these two facts are not true as the following two examples tell.

Example 3.15. Let $X=\{a, b, c\}, \tau=\{X, \phi,\{a, b\},\{c\}\}, \tau^{*}=\{X, \phi,\{a, c\}\}$. Then the identity function from $(X, \tau)$ onto $\left(X, \tau^{*}\right)$ is somewhat continuous; it is, however, not semicontinuous.

Example 3.16. Let $X=\{a, b, c, d\}, \tau=\{X, \phi,\{b\},\{d\},\{b, d\},\{a, d\},\{a, b, d\}\}, \tau^{*}=\{X, \phi$, $\{a, b\}\}$. Then the identity function from $(X, \tau)$ onto $\left(X, \tau^{*}\right)$ is even somewhat continuous and thus somewhat precontinuous; it is, however, not semi-precontinuous since $\{a, b\}$ is not semi-preopen in $(X, \tau)$.

The following result is a slight improvement of Proposition 3.10(i), the similar proof follows from Theorem 2.5 and the fact that if $A$ is a semiopen subset of a space $X$, then $\operatorname{pcl}(A)=\bar{A}$.

Proposition 3.17. (i) Let $f: X \rightarrow Y$ be a somewhat continuous and wrc-continuous function. If $X$ is almost $r c$-Lindelöf, then $Y$ is almost $r c$-Lindelöf.

(ii) Let $f: X \rightarrow Y$ be a somewhat precontinuous and weakly $\theta$-irresolute function. If $X$ is almost rc-Lindelöf, then $Y$ is almost rc-Lindelöf.

Remark 3.18. Clearly, every somewhat continuous function is somewhat precontinuous and every weakly $\theta$-irresolute function is wrc-continuous. However, the following two examples show that the property of being both somewhat continuous and wrc-continuous 
and the property of being both somewhat precontinuous and weakly $\theta$-irresolute are independent.

Example 3.19. Let $X=\{a, b, c\}, \tau=\{X, \phi,\{a, b\}\}, \tau^{*}=\{X, \phi,\{a, c\}\}$. Then the identity function from $(X, \tau)$ onto $\left(X, \tau^{*}\right)$ is somewhat precontinuous and weakly $\theta$-irresolute; it is, however, not somewhat continuous.

Example 3.20. Let $X=\{a, b, c, d\}, \tau=\{X, \phi,\{a\},\{b, c\},\{d\},\{a, b, c\},\{a, d\},\{b, c, d\}\}, \tau^{*}=$ $\{X, \phi,\{a, b\},\{d\},\{a, b, d\}\}$. Then the identity function from $(X, \tau)$ onto $\left(X, \tau^{*}\right)$ is somewhat continuous and wrc-continuous; it is, however, not weakly $\theta$-irresolute (observe that $\{d, c\}$ is regular closed in $\left(X, \tau^{*}\right)$ but not semiopen in $\left.(X, \tau)\right)$.

The following result is a slight improvement of Proposition 3.10(ii), it is a direct consequence of Remark 3.14 and Proposition 3.17.

Corollary 3.21. (i) Let $f: X \rightarrow Y$ be a surjective, semicontinuous, and wrc-continuous function. If $X$ is almost $r c$-Lindelöf, then $Y$ is almost $r c$-Lindelöf.

(ii) Let $f: X \rightarrow Y$ be a surjective, semi-precontinuous, and weakly $\theta$-irresolute function. If $X$ is almost rc-Lindelöf, then $Y$ is almost rc-Lindelöf.

Corollary 3.22 [2]. Let $f: X \rightarrow Y$ be a somewhat continuous and wrc-continuous surjection, where $Y$ is a weak $P$-space. If $X$ is rc-Lindelöf, then $Y$ is rc-Lindelöf.

Corollary 3.22 is still true even if the function $f$ is not surjective.

\section{Product theorems}

In this section, we study some types of functions that inversely preserve the property of being an rc-Lindelöf (almost rc-Lindelöf) set. We mainly obtain some product theorems concerning rc-Lindelöf spaces.

Definition 4.1 [19]. A function $f$ from a space $X$ into a space $Y$ is said to be regular open if it maps regular open subsets onto regular open subsets.

Definition 4.2 [19]. (i) A subset $A$ of a space $X$ is said to be an rc- $F_{\sigma}$ subset if $A$ is the countable union of regular closed subsets.

(ii) A function $f$ from a space $X$ into a space $Y$ is said to be weakly almost open if $f^{-1}(\bar{A}) \subset \overline{f^{-1}(A)}$ whenever $A$ is an rc- $F_{\sigma}$ subset of $Y$.

In [19], it was shown that every almost open function is weakly almost open, but not conversely.

Theorem 4.3 [19]. Let $f$ be a weakly almost open and regular open function from a space $X$ onto a space $Y$. Then the following hold.

(i) If for each $y \in Y, f^{-1}(y)$ is an S-set in $X$, then $X$ is almost $r c$-Lindelö whenever $Y$ is almost rc-Lindelöf.

(ii) If for each $y \in Y, f^{-1}(y)$ is $r c$-Lindelöf in $X$, then $X$ is almost $r c$-Lindelö $f$ whenever $Y$ is almost $r c$-Lindelof provided that $X$ is a weak $P$-space.

We point out here that in the result of Theorem 4.3(ii), $X$ being almost rc-Lindelöf may be replaced by rc-Lindelöf since $X$ is a weak $P$-space. 
Theorem 4.3 may be improved in the following form.

THeOREM 4.4. Let $f$ be a weakly almost open and regular open function from a space $X$ onto a space $Y$. Then the following hold.

(i) If for each $y \in Y, f^{-1}(y)$ is an S-set in $X$, then $f^{-1}(A)$ is almost rc-Lindelöf in $X$ whenever $A$ is almost $r$-Lindelö in $Y$.

(ii) If for each $y \in Y, f^{-1}(y)$ is rc-Lindelöf in $X$, then $f^{-1}(A)$ is rc-Lindelöf in $X$ whenever $A$ is almost $r c$-Lindelöf in $Y$ provided that $X$ is a weak $P$-space.

The following theorem shows that the assumption weakly almost open of Theorem 4.4 is not essential for the inverse preservation of the rc-Lindelöf set property.

Theorem 4.5. Let $f$ be a regular open function from a space $X$ onto a space $Y$. Then the following hold.

(i) If for each $y \in Y, f^{-1}(y)$ is an S-set in $X$, then $f^{-1}(A)$ is rc-Lindelof in $X$ whenever $A$ is rc-Lindelöf in $Y$.

(ii) If for each $y \in Y, f^{-1}(y)$ is rc-Lindelöf in $X$, then $f^{-1}(A)$ is rc-Lindelöf in $X$ whenever $A$ is rc-Lindelof in $Y$ provided that $X$ is a weak $P$-space.

The proof of the following proposition is straightforward and thus omitted.

Proposition 4.6. Let $X$ be a nearly Lindelöf space and $Y$ a weak $P$-space. Then the projection function $p: X \times Y \rightarrow Y$ sends regular closed sets onto closed sets.

Corollary 4.7. Let $X, Y$ be two spaces such that $Y$ is rc-Lindelöf and $X \times Y$ is extremally disconnected. Then the following hold.

(i) If $X$ is compact, then $X \times Y$ is rc-Lindelö [2].

(ii) If $X$ is Lindelöf, then $X \times Y$ is rc-Lindelöf provided that $X \times Y$ is a weak P-space.

Proof. We will show (ii), the other part is similar. Consider the projection function $p$ : $X \times Y \rightarrow Y$. Since $X \times Y$ is a weak $P$-space, it follows that $Y$ is a weak $P$-space, but $X$ is Lindelöf and thus nearly Lindelöf, so by Proposition 4.6, $p: X \times Y \rightarrow Y$ sends regular closed sets onto closed sets, but $X \times Y$ is extremally disconnected, so every regular open subset of $X \times Y$ is regular closed and thus $p: X \times Y \rightarrow Y$ sends regular open sets onto closed sets, but $p$ is an open function, so $p$ is regular open. Also for each $y \in Y, p^{-1}(y)=$ $X \times\{y\}$ is rc-Lindelöf in $X \times Y$ (as $X$ is Lindelöf and $X \times Y$ is extremally disconnected). Finally, since $Y$ is rc-Lindelöf, it follows immediately from Theorem 4.5(ii) that $X \times Y$ is rc-Lindelöf.

The following result is an improvement of Corollary 4.7, it follows from Theorem 1.2, Proposition 1.4, Corollary 4.7, and the fact that the properties of being extremally disconnected (a weak $P$-space) are hereditary with respect to open subsets.

Corollary 4.8. Let $X, Y$ be two rc-Lindelöf spaces such that $X \times Y$ is extremally disconnected. Then the following hold.

(i) If $X$ is locally compact, that is, for each $x \in X$, there exists an open set $U_{x}$ containing $x$ such that $\bar{U}_{x}$ is compact, then $X \times Y$ is rc-Lindelöf.

(ii) If $X$ is locally Lindelöf, that is, for each $x \in X$, there exists an open set $U_{x}$ containing $x$ such that $\overline{U_{x}}$ is Lindelöf, then $X \times Y$ is rc-Lindelöf provided that $X \times Y$ is a weak $P$-space. 


\section{Acknowledgment}

The author is grateful to the referee for his/her careful reading of the manuscript and for the valuable suggestions.

\section{References}

[1] M. E. Abd El-Monsef, S. N. El-Deeb, and R. A. Mahmoud, $\beta$-open sets and $\beta$-continuous mapping, Bulletin of the Faculty of Science. Assiut University. A. Physics and Mathematics 12 (1983), no. 1, 77-90.

[2] B. Al-Nashef and K. Al-Zoubi, A note on rc-Lindelof and related spaces, Questions and Answers in General Topology 21 (2003), no. 2, 159-170.

[3] D. Andrijević, Semi-preopen sets, Matematichki Vesnik 38 (1986), no. 1, 24-32.

[4] _ On b-open sets, Matematichki Vesnik 48 (1996), no. 1-2, 59-64.

[5] G. Balasubramanian, On some generalizations of compact spaces, Glasnik Matematički. Serija III 17(37) (1982), no. 2, 367-380.

[6] H. H. Corson and E. Michael, Metrizability of certain countable unions, Illinois Journal of Mathematics 8 (1964), 351-360.

[7] G. Di Maio, S-closed spaces, S-sets and S-continuous functions, Atti della Accademia delle Scienze di Torino 118 (1984), no. 3-4, 125-134.

[8] G. Di Maio and T. Noiri, On s-closed spaces, Indian Journal of Pure and Applied Mathematics 18 (1987), no. 3, 226-233.

[9] K. Dlaska, $r c$-Lindelöf sets and almost $r c$-Lindelöf sets, Kyungpook Mathematical Journal 34 (1994), no. 2, 275-281.

[10] K. Dlaska and M. Ganster, Almost rc-Lindelöf spaces, Bulletin of the Malaysian Mathematical Sciences Society. Second Series 17 (1994), 51-56.

[11] R. Engelking, General Topology, Sigma Series in Pure Mathematics, vol. 6, Heldermann, Berlin, 1989.

[12] Z. Frolík, Remarks concerning the invariance of Baire spaces under mappings, Czechoslovak Mathematical Journal 11 (86) (1961), 381-385.

[13] M. Ganster, T. Noiri, and I. L. Reilly, Weak and strong forms of $\theta$-irresolute functions, Journal of Institute of Mathematics \& Computer Sciences. (Mathematics Series) 1 (1988), no. 1, 19-29.

[14] D. S. Janković, A note on mappings of extremally disconnected spaces, Acta Mathematica Hungarica 46 (1985), no. 1-2, 83-92.

[15] D. S. Janković and C. Konstadilaki, On covering properties by regular closed sets, Mathematica Pannonica 7 (1996), no. 1, 97-111.

[16] N. Levine, Semi-open sets and semi-continuity in topological spaces, The American Mathematical Monthly 70 (1963), no. 1, 36-41.

[17] A. S. Mashhour, M. E. Abd El-Monsef, and S. N. El-Deep, On precontinuous and weak precontinuous mappings, Proceedings of the Mathematical and Physical Society of Egypt (1982), no. 53, 47-53 (1983).

[18] T. K. Mukherji and M. Sarkar, On a class of almost discrete spaces, Matematichki Vesnik 3(16)(31) (1979), no. 4, 459-474.

[19] M. S. Sarsak, On almost rc-Lindelöf sets, Acta Mathematica Hungarica 100 (2003), no. 1-2, 1-7.

[20] A. Wilansky, Topics in Functional Analysis, Lecture Notes in Mathematics, no. 45, Springer, Berlin, 1967.

Mohammad S. Sarsak: Department of Mathematics, Faculty of Science, The Hashemite University, P.O. Box 150459, Zarqa 13115, Jordan

E-mail address: sarsak@hu.edu.jo 


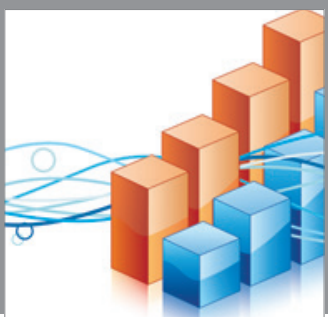

Advances in

Operations Research

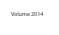

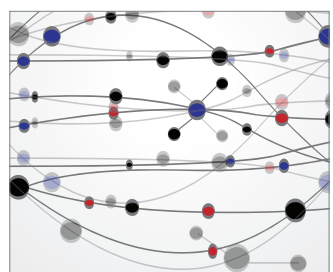

\section{The Scientific} World Journal
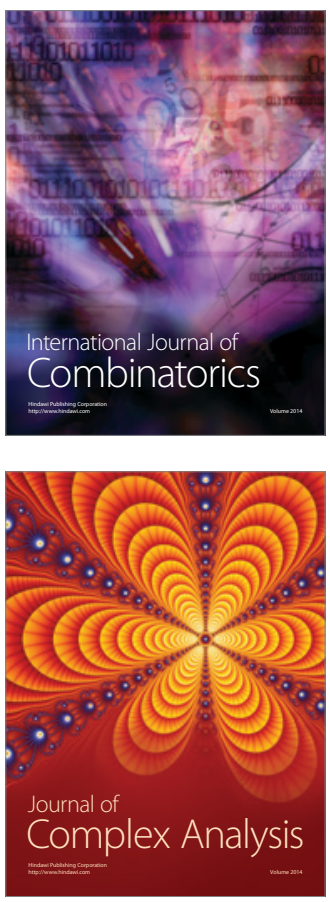

International Journal of

Mathematics and

Mathematical

Sciences
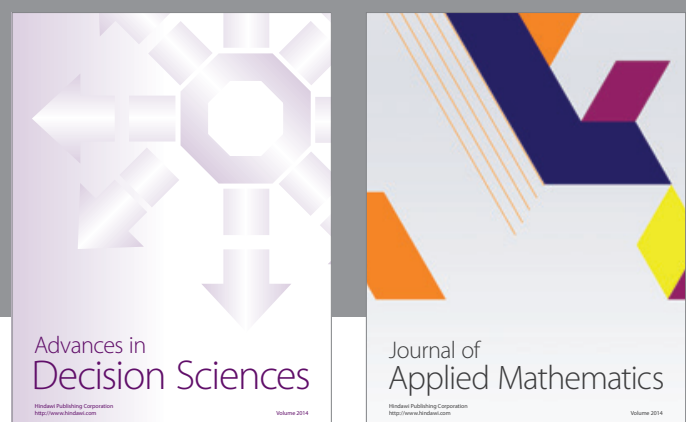

Journal of

Applied Mathematics
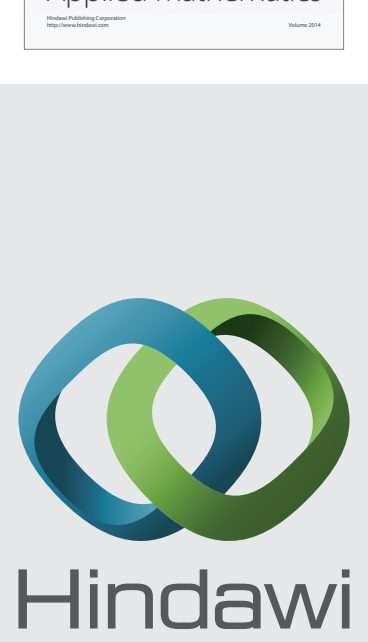

Submit your manuscripts at http://www.hindawi.com
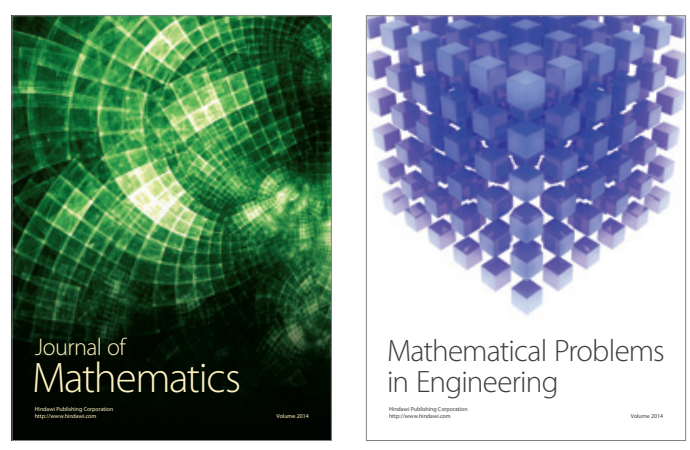

Mathematical Problems in Engineering
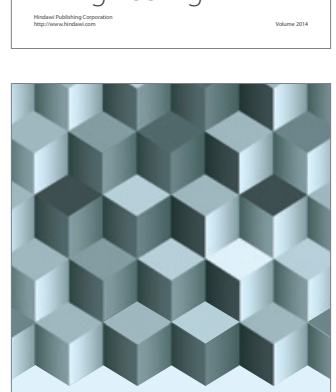

Journal of

Function Spaces
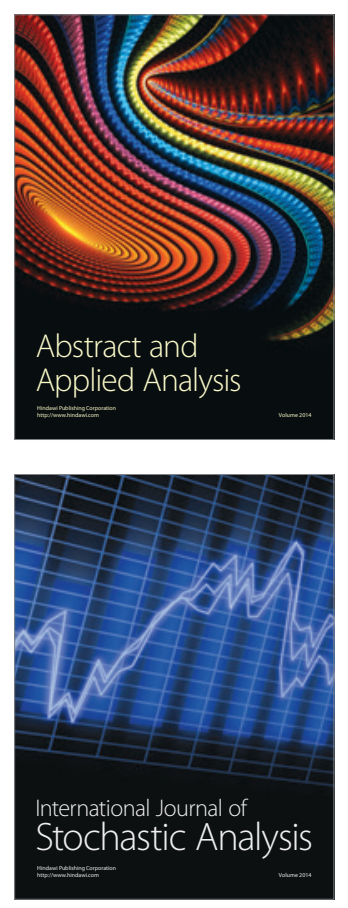

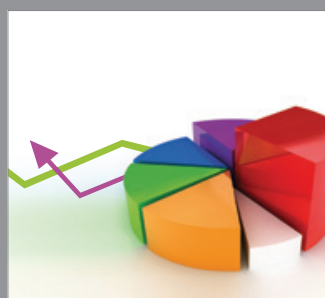

ournal of

Probability and Statistics

Promensencen
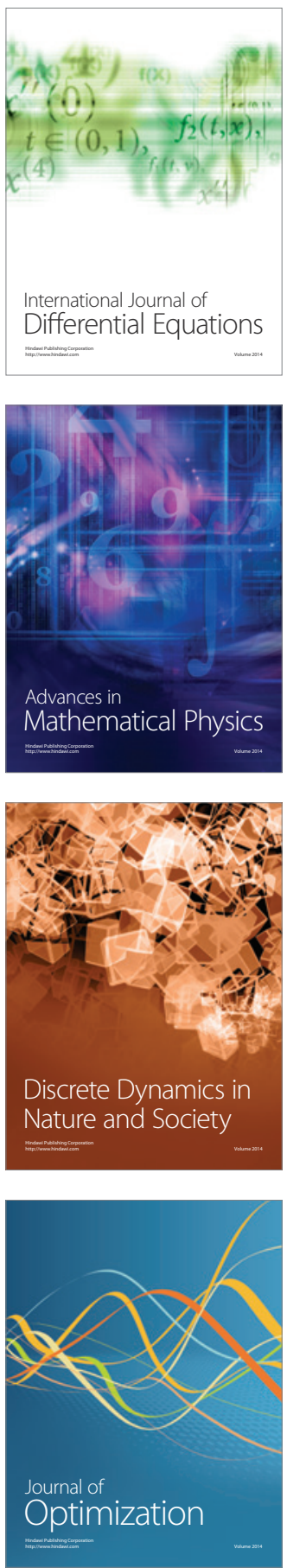